\title{
CONFÉRENCE
}

\section{Les agénésies dentaires : prise en charge en implantologie.}

\author{
Dr Lauwers L1, Pr Raoul G, Dr Rakotomalala H, Pr Ferri J
}

1. CHU Lille

Les agénésies dentaires sont à l'origine de répercussions importantes sur l'os alvéolaire et son développement. Une répercussion sur la croissance des bases osseuses est également présente à des degrés variables.

Notre expérience dans la prise en charge des agénésies dentaires, mais également de nombreux cas d'oligodontie, nous a permis de mettre en évidence les particularités cliniques et radiologiques, rencontrées chez ces patients. En fonction des localisations fréquemment retrouvées, nous guidons la stratégie de prise en charge globale à visée implantaire. De la greffe d'apposition dans le secteur incisif, à la dérivation nerveuse à la mandibule, ces solutions thérapeutiques sont évoquées dès le stade de l'imagerie tridimensionnelle de diagnostique.

Afin de préserver les tissus, nous « pilotons » les avulsions de dents lactéales, en relation avec le traitement orthodontique et chirurgical pré-implantaire.

Pendant la phase orthodontique, nous continuons à suivre les jeunes patients en consultation pluridisciplinaire afin d'échanger avec le praticien spécialisé sur nos souhaits de positions dentaires, modification de formule, d'alignement, d'espaces implantaires, afin de contrôler que ceux ci sont en adéquation avec la finalité prothétique et implantaire.

En fin de croissance, nous débutons notre prise en charge implantaire. Elle comporte dans la très grande majorité des cas, des greffes osseuses, associées si nécessaire à une chirurgie orthognatique, ou autre technique chirurgicale. Tout l'éventail des interventions de chirurgie maxillo-faciale est disponible. II est alors question en fin de cette croissance pilotée par le traitement orthodontique et les prothèses adjointes, de positionner idéalement les bases osseuses dans les trois sens de l'espace et de recréer tout le volume osseux nécessaire à la mise en place d'implant en nombre, longueur et diamètre optimaux. Après un délai de consolidation, nous envisageons la planification implantaire en accord avec le patient et le projet initial.

Nous pratiquons le plus souvent possible, la technique d'extraction « dent lactéale » implantation immédiate, car elle nous permet de préserver les volumes osseux, mais également de mieux gérer les tissus mous dans les secteurs esthétiques. En Effet, même si cette démarche complique le déroulement du plan de traitement, sans toutefois le compromettre, elle se justifie de notre point de vue par une amélioration des résultats de tissus péri-implantaires et notamment de qualité de gencive attachée.

Les échanges avec les différentes disciplines de notre exercice sont toujours bénéfiques à nos patients, pour la réussite du traitement, mais également pour le simplifier, tout en évitant les compromis.

lauwers.ludovic@gmail.com

(C) The authors, published by EDP Sciences. This is an Open Access article distributed under the terms of the Creative Commons Attribution License 4.0 (http://creativecommons.org/licenses/by/4.0/). 